\title{
Fitting Conics to Noisy Data Using Stochastic Linearization
}

\author{
Marcus Baum and Uwe D. Hanebeck
}

\begin{abstract}
Fitting conic sections, e.g., ellipses or circles, to noisy data points is a fundamental sensor data processing problem, which frequently arises in robotics. In this paper, we introduce a new procedure for deriving a recursive Gaussian state estimator for fitting conics to data corrupted by additive Gaussian noise. For this purpose, the original exact implicit measurement equation is reformulated with the help of suitable approximations as an explicit measurement equation corrupted by multiplicative noise. Based on stochastic linearization, an efficient Gaussian state estimator is derived for the explicit measurement equation. The performance of the new approach is evaluated by means of a typical ellipse fitting scenario.
\end{abstract}

\section{INTRODUCTION}

In this work, the problem of fitting a conic section such as an ellipse or a circle to noisy data points is considered. This fundamental problem arises in many applications related to robotics. A traditional application is computer vision, where ellipses and circles are fitted to features extracted from images [1]-[4] in order to detect, localize, and track objects. For instance in [5], [6], ellipse fitting methods are used by a humanoid robot for localizing objects. Apart from image data, conic fitting algorithms are also suitable for data obtained from laser or radar devices. For example, mobile robots use circle fitting algorithms for outdoor localization based on circular landmarks [7], [8] measured with a laser range scanner and in [9], [10], ellipse fits are used for people tracking based on a laser scanner (see Fig. 1).

The huge variety of application areas has led to many different solution methodologies for conic fitting because each application has its specific requirements on the fitting algorithm [11]. For instance, if data is extracted from an image, the noise is typically rather small and a large number of measurements is available at a batch. However, a laser scanner or a radar device usually yields measurements with high measurement noise and only a few measurements are available per scan.

In this paper, we focus on tracking applications in which the measurements are received sequentially and the parameters of the conic may evolve over time. Each received measurement is assumed to stem from an unknown measurement source on the conic and is corrupted by additive Gaussian noise. The statistics of the measurement noise are a priori known as they result from a particular sensor such as a laser scanner. As common for tracking applications, we seek a recursive Bayesian state estimator [12] that recursively incorporates measurements by updating a probability

Marcus Baum and Uwe D. Hanebeck are with the Intelligent Sensor-Actuator-Systems Laboratory (ISAS), Institute for Anthropomatics, Karlsruhe Institute of Technology (KIT), Germany. marcus.baumakit.edu, uwe. hanebecklieee.org

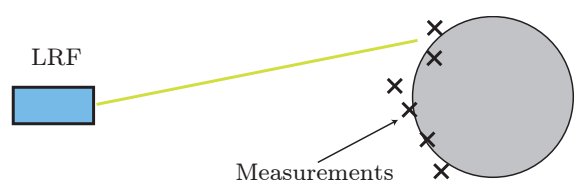

Fig. 1: Laser rangefinder (LRF) scanning a circular object.

distributions for the parameter vector of the conic based on Bayes' rule. An explicit description of the parameter uncertainties with a probability distribution is in particular important for performing gating, i.e., dismissing unprobable measurements, and tracking multiple conics. Furthermore, the temporal evolution of the state can be captured with a stochastic system model, which allows to propagate the uncertainty about the state to the next time step.

\section{A. Contributions}

In this paper, we present a new method for deriving recursive Gaussian-assumed Bayesian state estimators for fitting a conic to data corrupted by additive Gaussian noise. For this purpose, we show how the original implicit measurement equation can be approximated with an explicit quadratic measurement equation that is corrupted by multiplicative Gaussian noise. Based on this measurement equation, a standard nonlinear Gaussian state estimator that performs stochastic linearization can be employed for state estimation. It is also possible to derive analytic expressions based on analytic moment calculation for optimal stochastic linearization. The performance of the new approach is demonstrated by simulations.

\section{B. Related Work}

As curve fitting is an old fundamental problem, many different solution methodologies exist. For instance, nonBayesian methods for conic fitting are the algebraic fit [13], [14] and the geometric fit [15]. Statistical methods such as maximum-likelihood estimation are discussed in [11], [16], [17]. In [18], fitting problems are formulated as a hierarchical probability model, i.e., the measurement sources are assumed to be drawn from a particular probability distributions.

The standard Bayesian approach for tracking conic sections is based on the Extended Kalman Filter (EKF) [1], [2] for implicit measurement equations. This approach linearizes the implicit conic equation around the current estimate and the measurement in order to obtain an explicit linear measurement equation. Based on the linear measurement equation, a Gaussian density for the conic parameters can be recursively updated with the Kalman filter equations. This 


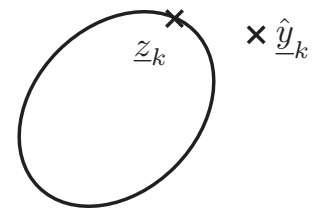

Fig. 2: Illustration of the measurement source $\underline{z}_{k}$ and the measurement $\underline{\hat{y}}_{k}$ for an ellipse.

approach comes with two major disadvantages regarding the considered application here: First, as the implicit conic equation is linearized around the measurement, the linearization error may become high in case of outliers and, hence, unstable and poor estimates are obtained in case of high measurement noise. Furthermore, the representation of an ellipse used in [1], [2] is unsuitable for tracking applications because the center of the ellipse is not directly contained in the parameter vector. However, the dynamics are usually expressed as a linear equation depending on the ellipse center.

In [19], the authors of this paper have derived a Bayesian method for fitting circles if data with isotropic noise is given. The relationship of [19] to the work described here in this paper is discussed in Section IV-A.4.

\section{Problem Formulation}

In this work, the problem is to estimate the time-varying parameter vector of a conic ${ }^{1} \underline{\boldsymbol{p}}_{k}$, where $k$ denotes the time index, based on sequentially arriving measurements $\underline{\hat{y}}_{k}$.

A conic is a quadric in the Euclidean plane. A quadric is a hypersurface in $n$-dimensional space that is specified by the solution of an implicit quadratic polynomial, i.e.,

$$
\left\{\underline{z} \in \mathbb{R}^{n} \mid g\left(\underline{\boldsymbol{p}}_{k}, \underline{z}\right)=0\right\},
$$

where $\underline{\boldsymbol{p}}_{k}$ are the parameters of the quadric and

$$
g\left(\underline{\boldsymbol{p}}_{k}, \underline{z}\right)=\underline{z}^{T} \mathbf{A}_{k} \underline{z}+\underline{\boldsymbol{b}}_{k}^{T} \underline{z}+\boldsymbol{f}_{k},
$$

with $n \times n$-dimensional matrix $\mathbf{A}_{k}, n$-dimensional vector $\underline{\boldsymbol{b}}_{k}$, and constant $\boldsymbol{f}_{k}$. Note that the parameter vector $\underline{\boldsymbol{p}}_{k}$ is a suitable representation of $\mathbf{A}_{k}, \underline{b}_{k}$, and $\boldsymbol{f}_{k}$.

Example II.1 A conic section and can be parameterized by the parameter vector $\underline{\boldsymbol{p}}_{k}=\left[\boldsymbol{a}_{k}, \boldsymbol{b}_{k}, \boldsymbol{c}_{k}, \boldsymbol{d}_{k}, \boldsymbol{e}_{k}, \boldsymbol{f}_{k}\right]^{T}$, where $\mathbf{A}=\left[\begin{array}{ll}\boldsymbol{a}_{k} & \boldsymbol{c}_{k} \\ \boldsymbol{c}_{k} & \boldsymbol{b}_{k}\end{array}\right]$, and $\underline{\boldsymbol{b}}_{k}=\left[\begin{array}{l}\boldsymbol{d}_{k} \\ \boldsymbol{e}_{k}\end{array}\right]$. An ellipse is a special conic section (1) that can be described by five parameters. We will discuss proper representations for ellipses in the following section.

In order to estimate $\underline{\boldsymbol{p}}_{k}$, a measurement model that relates the state to measurements and a dynamic model for the temporal evolution of the state is given as described in the following.

\footnotetext{
${ }^{1}$ We focus on the two-dimensional case, i.e., conic sections. However, the results are directly applicable for fitting a quadric in $n$-dimensional space.
}

\section{A. Measurement Model}

The measurement model relates the state vector $\underline{\boldsymbol{p}}_{k}$ to the measurement $\underline{\hat{y}}_{k}$. We assume that the measurement $\underline{\hat{y}}_{k}$ is the noisy observation of a so-called measurement source $\underline{z}_{k}$ according to

$$
\underline{\hat{y}}_{k}=\underline{z}_{k}+\underline{\boldsymbol{v}}_{k}
$$

where the noise term $\underline{\boldsymbol{v}}_{k}$ is zero-mean white Gaussian noise with known covariance matrix $\Sigma_{k}^{v}$ (see Fig. 2). The measurement source $\underline{z}_{k}$ is known to lie on the conic section, i.e.,

$$
g\left(\underline{\boldsymbol{p}}_{k}, \underline{z}_{k}\right)=0 .
$$

It is important to note that no further information about the measurement source $\underline{z}_{k}$ on the conic section is given, e.g., no specific probability distribution for $\underline{z}_{k}$ is given. Hence, (3) and (4) specify an implicit measurement equation. Such a model is also known as Errors-In-Variables Model [11]. Note that we are not interested in the location of the measurement source itself, so it is not a part of the state vector.

\section{B. Dynamic Model}

The temporal evolution of the parameter vector $\underline{p}_{k}$ may be given by a stochastic dynamic model, which can be characterized by a system equation in the form

$$
\underline{\boldsymbol{p}}_{k+1}=a_{k}\left(\underline{\boldsymbol{p}}_{k}, \underline{u}_{k}, \underline{\boldsymbol{w}}_{k}\right),
$$

where $a_{k}(\cdot)$ is the system function, $\underline{u}_{k}$ is the system input, and $\underline{\boldsymbol{w}}_{k}$ the system noise.

\section{Bayesian State Estimator}

In this work, a recursive Gaussian state estimator for estimating the parameters of the conic is desired. A Bayesian state estimator recursively computes a probability density for the state by alternating a time update and a measurement update. A Gaussian state estimator represents the uncertainty about $\underline{\boldsymbol{p}}_{k}$ having received the measurements $\underline{\hat{y}}_{1}, \ldots, \underline{\hat{y}}_{k}$ with a Gaussian density $f^{e}\left(\underline{p}_{k}\right)=\mathcal{N}\left(\underline{p}_{k}-\underline{\mu}_{k}^{e}, \Sigma_{k}^{e}\right)$, where $\underline{\mu}_{k}^{e}$ is the mean and $\Sigma_{k}^{\bar{e}}$ is the covariance matrix. The prediction for time step $k+1$ having received measurements $\underline{\hat{y}}_{1}, \ldots, \hat{y}_{k}$ is represented with the Gaussian density $f^{p}\left(\underline{p}_{k+1}\right)=\mathcal{\mathcal { N }}\left(\underline{p}_{k+1}-\underline{\mu}_{k+1}^{p}, \Sigma_{k+1}^{p}\right)$ with mean $\underline{\mu}_{k+1}^{p}$ and
covariance matrix $\Sigma_{k+1}^{p}$.

Because only an implicit measurement model as described in Section II-A is available, the standard Bayesian formalism cannot be directly applied to this problem. We suggest an approximation method for rewriting the implicit measurement model to an explicit model in Section IV such that standard Bayesian filtering techniques can be applied.

\section{Parameterizing Conic Section}

In this section, we discuss suitable parameterizations of ellipses and circles as they are the most relevant conic sections for practical applications. In two-dimensional space, a general conic section as given in (2) is described by six parameters and (2) describes an ellipse if $\mathbf{A}_{k}$ has only positive eigenvalues. Usually, it is difficult to incorporate this constraint in the fitting algorithm. For instance, directly using 
the representation of Example II.1 can cause problems as the representation with a Gaussian density does not capture this constraint, e.g., the mean may not describe a valid ellipse.

In the following, we discuss two alternative representations for ellipses that aim at avoiding this problem by using five parameters for describing an ellipse. These representations are special cases of (2).

Ellipse (Coefficient Representation): A widely-used representation of an ellipse (see [1], [2], [20]) results from writing (2) as

$$
\boldsymbol{a}_{k} x^{2}+2 \boldsymbol{b}_{k} x y+\boldsymbol{c}_{k} y^{2}+2 \boldsymbol{d}_{k} x+2 \boldsymbol{e}_{k} y+\boldsymbol{f}_{k}=0,
$$

where $\underline{z}=[x, y]$. Because $\boldsymbol{a}_{k}+\boldsymbol{c}_{k} \neq 0$ for an ellipse, one parameter can be removed by assuming $\boldsymbol{a}_{k}+\boldsymbol{c}_{k}=1$. An ellipse is then given by the five-dimensional parameter vector $\underline{\boldsymbol{p}}_{k}=\left[\boldsymbol{a}_{k}, \boldsymbol{b}_{k}, \boldsymbol{d}_{k}, \boldsymbol{e}_{k}, \boldsymbol{f}_{k}\right]^{T}$ and the implicit equation

$$
\boldsymbol{a}_{k}\left(x^{2}-y^{2}\right)+2 \boldsymbol{b}_{k} x y+y^{2}+2 \boldsymbol{d}_{k} x+2 \boldsymbol{e}_{k} y+\boldsymbol{f}_{k}=0 .
$$

Note that the obvious normalization $\boldsymbol{f}_{k}=1$ would exclude ellipses through the origin.

Although the coefficient representation of an ellipse is widely used, it comes with two major disadvantages in the application considered here: First, there still are parameters vectors that do not describe an ellipse. While in many applications such as computer vision, this not a relevant problem as enough data is available to exclude infeasible solutions, the situation may be different in tracking applications. Due to rather high measurement noise, less information about an ellipse is available at a particular point in time and hence, infeasible parameter estimates may be frequently obtained. Furthermore, the dynamic model is usually described in terms of the center of the ellipse. However, the center of the ellipse is not directly available from the parameters and hence, the dynamic models for this kind of parametric representation become quite complicated, i.e., nonlinear and unintuitive. As a consequence, we suggest a different parameterization of an ellipse, as discussed in the following.

Ellipse (Center/Shape Representation): An $n$-dimensional ellipsoid can be represented by the center $\underline{\boldsymbol{m}}_{k}$ and a positive semi-definite shape matrix $\mathbf{B}_{k}$ according to

$$
\left\{\underline{z} \mid \underline{z} \in \mathbb{R}^{n} \text { and }\left(\underline{z}-\underline{\boldsymbol{m}}_{k}\right)^{T} \mathbf{B}_{k}^{-1}\left(\underline{z}-\underline{\boldsymbol{m}}_{k}\right)-1=0\right\} .
$$

The positive semi-definite matrix $\mathbf{B}_{k}^{-1}$ can be represented with its Cholesky decomposition $\mathbf{B}_{k}^{-1}=\mathbf{L}_{k} \mathbf{L}_{k}^{T}$, where

$$
\mathbf{L}_{k}:=\left[\begin{array}{cc}
\boldsymbol{l}_{k}^{(1)} & 0 \\
\boldsymbol{l}_{k}^{(3)} & \boldsymbol{l}_{k}^{(2)}
\end{array}\right]
$$

is a lower triangular matrix with positive diagonal entries. The parameter vector $\underline{\boldsymbol{p}}_{k}$ of an ellipse is then given by $\underline{\boldsymbol{p}}_{k}=\left[\underline{\boldsymbol{m}}_{k}^{T}, \boldsymbol{l}_{k}^{(1)}, \boldsymbol{l}_{k}^{(2)}, \boldsymbol{l}_{k}^{(3)}\right]^{T}$, which consists of the center and the non-zero entries of the Cholesky decomposition. The quadratic function (1) then becomes

$$
g\left(\underline{\boldsymbol{p}}_{k}, \underline{z}\right):=\left(\underline{z}-\underline{\boldsymbol{m}}_{k}\right)^{T} \cdot\left(\mathbf{L}_{k} \mathbf{L}_{k}^{T}\right) \cdot\left(\underline{z}-\underline{\boldsymbol{m}}_{k}\right)-1 .
$$

The above parameterization explicitly contains the ellipse center and a valid ellipse is specified by $\mathbf{L}_{k} \mathbf{L}_{k}^{T}$. Furthermore, it can directly be used for higher-dimensional ellipsoids. Note that this representation has already been used by the authors for approximating the shape of groups and extended objects [21]. Further specific conic sections are circles, which are relevant for practical applications [11].

Circle (Center/Radius Representation): An obvious parameterization of a circle in two-dimensional space is given by $\underline{\boldsymbol{p}}_{k}=\left[\underline{\boldsymbol{m}}_{k}^{T}, \boldsymbol{r}_{k}\right]^{T}$, where $\underline{\boldsymbol{m}}_{k}=\left[\boldsymbol{m}_{k, 1}, \boldsymbol{m}_{k, 2}\right]^{T}$ is the center and $\boldsymbol{r}_{k}$ the radius. The quadratic function in (1) then becomes $g\left(\underline{\boldsymbol{p}}_{k}, \underline{z}\right)=\left(x-\boldsymbol{m}_{k, 1}\right)^{2}+\left(y-\boldsymbol{m}_{k, 2}\right)^{2}-\boldsymbol{r}_{k}^{2}$.

\section{Conic Fitting Based on Stochastic LINEARIZATION}

In this section, we introduce a new method for conic fitting based on a Gaussian state estimator.

\section{A. Measurement Update}

In order to derive an explicit measurement equation, we use the general form of a conic section (2). Based on this derivation, the particular example representations of conics introduced in Section III are treated in Section IV-A.4.

The first step is to put (3) in (4), i.e.,

$$
\begin{aligned}
g\left(\underline{\boldsymbol{p}}_{k}, \underline{\hat{y}}_{k}\right)= & g\left(\underline{\boldsymbol{p}}_{k}, \underline{z}_{k}+\underline{\boldsymbol{v}}_{k}\right) \\
= & g\left(\underline{\boldsymbol{p}}_{k}, \underline{z}_{k}\right) \\
& +2 \underline{z}_{k}^{T} \mathbf{A}_{k} \underline{\boldsymbol{v}}_{k}+\underline{\boldsymbol{v}}_{k}^{T} \mathbf{A}_{k} \underline{\boldsymbol{v}}_{k}+\underline{\boldsymbol{b}}_{k}^{T} \underline{\boldsymbol{v}}_{k} .
\end{aligned}
$$

Because it follows from (4) that $g\left(\underline{\boldsymbol{p}}_{k}, \underline{z}_{k}\right)=0$, the following equation is obtained

$$
0=g\left(\underline{\boldsymbol{p}}_{k}, \underline{\hat{y}}_{k}\right)-\left(2 \underline{z}_{k}^{T} \mathbf{A}_{k} \underline{\boldsymbol{v}}_{k}+\underline{\boldsymbol{v}}_{k}^{T} \mathbf{A}_{k} \underline{\boldsymbol{v}}_{k}+\underline{\boldsymbol{b}}_{k}^{T} \underline{\boldsymbol{v}}_{k}\right) .
$$

Based on (8), a measurement equation with multiplicative noise and a measurement equation with additive noise can be derived.

1) Measurement Equation with Multiplicative Noise: Equation (8) can directly interpreted as an explicit measurement equation of the form

$$
0=h\left(\underline{\boldsymbol{p}}_{k}, \underline{\boldsymbol{v}}_{k}\right),
$$

where $h\left(\underline{\boldsymbol{p}}_{k}, \underline{\boldsymbol{v}}_{k}\right)$ is a new measurement function that maps the random variables $\underline{\boldsymbol{p}}_{k}$ and $\underline{\boldsymbol{v}}_{k}$ to a pseudo-measurement with value 0 . It is important to note that $h\left(\underline{\boldsymbol{p}}_{k}, \underline{\boldsymbol{v}}_{k}\right)$ also depends on the measurement $\underline{y}_{k}$ and the unknown measurement source $\underline{z}_{k}$. The basic idea is to substitute $\underline{z}_{k}$ in (8) by a proper point estimate. How this point estimate for $\underline{z}_{k}$ is obtained is discussed in Section IV-A.3.

Although the measurement equation is nonlinear and nonadditive Gaussian noise is involved, a measurement update can be performed with basic filtering techniques because only quadratic nonlinearities occur. For instance, a measurement update can be performed with a Gaussian filter such as the UKF [22], which approximates the first two moments of the joint state of the state and the measurement with a 
Gaussian distribution (this process is also called stochastic linearization).

Given the prediction $f^{p}\left(\underline{p}_{k}\right)=\mathcal{N}\left(\underline{p}_{k}-\underline{\mu}_{k}^{p}, \Sigma_{k}^{p}\right)$, a measurement update with the measurement $\underline{\hat{y}}_{k}$ can be performed according to the Kalman filter [23] equations

$$
\begin{aligned}
& \underline{\mu}_{k}^{e}=\underline{\mu}_{k}^{p}+\Sigma_{k}^{p h}\left(\Sigma_{k}^{h h}\right)^{-1}\left(0-\underline{\mu}_{k}^{h}\right), \\
& \Sigma_{k}^{e}=\Sigma_{k}^{p}-\Sigma_{k}^{p h}\left(\Sigma_{k}^{h h}\right)^{-1} \Sigma_{k}^{h p},
\end{aligned}
$$

where 0 is the predicted pseudo-measurement, $\Sigma_{k}^{p h}$ is covariance between $h\left(\underline{\boldsymbol{p}}_{k}, \underline{\boldsymbol{v}}_{k}\right)$ and $\underline{\boldsymbol{p}}_{k}$, and $\sum_{k}^{h h}$ is the variance of $h\left(\underline{\boldsymbol{p}}_{k}, \underline{\boldsymbol{v}}_{k}\right)$. At this point it is important to note that $\underline{\mu}_{k}^{h}$ and $\Sigma_{k}^{p h}$ do not depend on the unknown measurement source $\underline{z}_{k}$ and, hence, the error made due to substituting it with a point estimate is rather negligible. A measurement update as described above can be performed with a Gaussian state estimator such as the UKF [22] or even based on analytic moment calculation as described in [24]. Note that also nonGaussian estimators could be used for obtaining even more precise estimation results.

2) Measurement Equation with Additive Noise: Equation (8) can also be used for constructing a measurement equation with additive Gaussian noise, which is easier to handle with many nonlinear filters, such as particle filters or the EKF [23]. For this purpose, (8) is written as

$$
0=g\left(\underline{\boldsymbol{p}}_{k}, \underline{\hat{y}}_{k}\right)+\bar{v}_{k}\left(\underline{\boldsymbol{p}}_{k}, \underline{\boldsymbol{v}}_{k}\right),
$$

where $\bar{v}_{k}\left(\underline{\boldsymbol{p}}_{k}, \underline{\boldsymbol{v}}_{k}\right)$ is a noise term that depends on the shape parameters $\underline{\boldsymbol{p}}_{k}$, the measurement noise $\underline{\boldsymbol{v}}_{k}$, but also on the unknown measurement source $\underline{z}_{k}$. In order to remove the dependency of $\bar{v}_{k}\left(\underline{\boldsymbol{p}}_{k}, \underline{\boldsymbol{v}}_{k}\right)$ on $\underline{\boldsymbol{p}}_{k}$, we substitute its current estimate $\underline{\mu}_{k}^{p}$ to obtain

$$
0=g\left(\underline{\boldsymbol{p}}_{k}, \underline{\hat{y}}_{k}\right)+\bar{v}_{k}\left(\underline{\mu}_{k}^{p}, \underline{\boldsymbol{v}}_{k}\right) .
$$

Again we have to substitute a point estimate for the unknown measurement source $\underline{z}_{k}$ as described in Section IV-A.3. The probability distribution of $\bar{v}_{k}\left(\underline{\mu}_{k}^{p}, \underline{\boldsymbol{v}}_{k}\right)$ can be approximated with a Gaussian density by means of nonlinear estimation techniques, e.g., [22], [24]. Note that it can directly be seen that the mean $\mathrm{E}\left\{\bar{v}_{k}\left(\underline{\mu}_{k}^{p}, \underline{\boldsymbol{v}}_{k}\right)\right\}$ does not depend on the measurement source.

3) Obtaining a Point Estimate for the Measurement Source: In the following, we show how a point estimate for the measurement source $\underline{z}_{k}$ can be obtained based on the prediction $f^{p}\left(\underline{p}_{k+1}\right)=\mathcal{N}\left(\underline{p}_{k+1}-\underline{\mu}_{k+1}^{e}, \Sigma_{k+1}^{e}\right)$ and the measurement $\underline{\hat{y}}_{k}$.

A simple method is to consider the conic specified by the mean of the prediction, i.e., $\underline{\mu}_{k+1}^{p}$, and use the point with the smallest distance from the conic to the measurement $\underline{\hat{y}}_{k}$ as a point estimate for the measurement source $\underline{z}_{k}$.

A more elaborate method is to find the most likely measurement source for given $\underline{\mu}_{k+1}^{p}$ and $\underline{\hat{y}}_{k}$.

4) Examples: Having explained the general procedure for deriving the explicit measurement equation for conic fitting, particular measurement equations are derived in the following for the ellipse and circle representations given in Section III.
Ellipse (Coefficient Representation): If the components of the measurement source are given by $\underline{z}_{k}=\left[x_{k}^{z}, y_{k}^{z}\right]^{T}$, and the measurement noise is denoted with $\underline{\boldsymbol{v}}_{k}=\left[\boldsymbol{v}_{k}^{x}, \boldsymbol{v}_{k}^{y}\right]^{T}$, (8) becomes

$$
\begin{aligned}
0= & g\left(\underline{\boldsymbol{p}}_{k}, \hat{y}_{k}\right)- \\
& \boldsymbol{a}_{k}\left(x_{k}^{z} \boldsymbol{v}_{k}^{x}+\left(\boldsymbol{v}_{k}^{x}\right)^{2}-y_{k}^{z} \boldsymbol{v}_{k}^{y}-\left(\boldsymbol{v}_{k}^{y}\right)^{2}\right) \\
& 2 \boldsymbol{b}_{k}\left(x_{k}^{z} \boldsymbol{v}_{k}^{y}+y_{k}^{z} \boldsymbol{v}_{k}^{x}+\boldsymbol{v}_{k}^{y} \boldsymbol{v}_{k}^{x}\right)+ \\
& y_{k}^{z} \boldsymbol{v}_{k}^{y}+\left(\boldsymbol{v}_{k}^{y}\right)^{2}+2 \boldsymbol{d}_{k} \boldsymbol{v}_{k}^{y}+2 \boldsymbol{e}_{k} \boldsymbol{v}_{k}^{x} \\
:= & h\left(\underline{\boldsymbol{p}}_{k}, \underline{\boldsymbol{v}}_{k}\right),
\end{aligned}
$$

where $h\left(\underline{\boldsymbol{p}}_{k}, \underline{\boldsymbol{v}}_{k}\right)$ is the new measurement equation with multiplicative noise. For the measurement equation with additive noise (9), the noise term becomes

$$
\begin{aligned}
& \bar{v}_{k}\left(\underline{\boldsymbol{p}}_{k}, \underline{\boldsymbol{v}}_{k}\right)= \boldsymbol{a}_{k}\left(x_{k}^{z} \boldsymbol{v}_{k}^{x}+\left(\boldsymbol{v}_{k}^{x}\right)^{2}-y_{k}^{z} \boldsymbol{v}_{k}^{y}-\left(\boldsymbol{v}_{k}^{y}\right)^{2}\right) \\
& \boldsymbol{b}_{k}\left(x_{k}^{z} \boldsymbol{v}_{k}^{y}+y_{k}^{z} \boldsymbol{v}_{k}^{x}+\boldsymbol{v}_{k}^{y} \boldsymbol{v}_{k}^{x}\right)+ \\
& y_{k}^{z} \boldsymbol{v}_{k}^{y}+\left(\boldsymbol{v}_{k}^{y}\right)^{2}+2 \boldsymbol{d}_{k} \boldsymbol{v}_{k}^{y}+2 \boldsymbol{e}_{k} \boldsymbol{v}_{k}^{x} .
\end{aligned}
$$

The probability distribution of $\bar{v}_{k}\left(\mu_{k}^{p}, \underline{\boldsymbol{v}}_{k}\right)$ can be approximated with a Gaussian distribution by means of analytic moment calculation as described in [24]. It is interesting to note that $g\left(\underline{\boldsymbol{p}}_{k}, \underline{\hat{y}}_{k}\right)$ is linear in $\underline{\boldsymbol{p}}_{k}$ and, hence, we end up with a linear measurement equation.

Ellipse (Center/Shape Representation): For this parameterization of an ellipse, (8) becomes

$$
\begin{aligned}
0 & =g\left(\underline{\boldsymbol{p}}_{k}, \hat{y}_{k}\right)-2\left(\underline{z}_{k}-\underline{\boldsymbol{m}}_{k}\right)^{T} \mathbf{B}_{k}^{-1} \underline{\boldsymbol{v}}_{k}+\underline{\boldsymbol{v}}_{k}^{T} \mathbf{B}_{k}^{-1} \underline{\boldsymbol{v}}_{k} \\
& :=h\left(\underline{\boldsymbol{p}}_{k}, \underline{\boldsymbol{v}}_{k}\right)
\end{aligned}
$$

where $h\left(\underline{\boldsymbol{p}}_{k}, \underline{\boldsymbol{v}}_{k}\right)$ is the new measurement function. ${ }^{2}$ The additive noise term in (9) is given by $\bar{v}_{k}\left(\underline{\boldsymbol{p}}_{k}, \underline{\boldsymbol{v}}_{k}\right)=2\left(\underline{z}_{k}-\right.$ $\left.\underline{\boldsymbol{m}}_{k}\right)^{T} \mathbf{B}_{k}^{-1} \underline{\boldsymbol{v}}_{k}+\underline{\boldsymbol{v}}_{k}^{T} \mathbf{B}_{k}^{-1} \underline{\boldsymbol{v}}_{k}$. The noise term $\bar{v}_{k}\left(\underline{\mu}_{k}^{p}, \underline{\boldsymbol{v}}_{k}\right)$ can be approximated with a Gaussian density by means of moment matching. The exact expressions are given in the following theorem.

Theorem IV.1 For given parameters $\underline{\mu}_{k}^{p}$ and the corresponding shape matrix $\hat{\mathbf{B}}_{k}$ and ellipse center $\underline{\hat{m}}_{k}$, the first two moments of the random variable $\bar{v}_{k}\left(\underline{\mu}_{k}^{p}, \underline{\boldsymbol{v}}_{k}\right)$ are given by

$$
\begin{array}{ll}
\text { - } & \mathrm{E}\left\{\bar{v}_{k}\left(\underline{\mu}_{k}^{p}, \underline{\boldsymbol{v}}_{k}\right)\right\}=\operatorname{trace}\left(\hat{\mathbf{B}}_{k}^{-1} \Sigma_{k}^{v}\right) \text {, and } \\
\text { - } & \operatorname{Var}\left\{\bar{v}_{k}\left(\underline{\mu}_{k}^{p}, \underline{\boldsymbol{v}}_{k}\right)\right\}=2 \operatorname{trace}\left(\hat{\mathbf{B}}_{k}^{-1} \Sigma_{k}^{v} \hat{\mathbf{B}}_{k}^{-1} \Sigma_{k}^{v}\right)+4\left(\underline{z}_{k}-\right. \\
& \left.\underline{\hat{m}}_{k}\right)^{T} \hat{\mathbf{B}}_{k}^{-1} \Sigma_{k}^{v} \hat{\mathbf{B}}_{k}^{-1}\left(\underline{z}_{k}-\underline{\hat{m}}_{k}\right) .
\end{array}
$$

PROOF. Follows from the formulas for quadratic forms of normal distributions in [25], [26].

The first moment $\mathrm{E}\left\{\bar{v}_{k}\left(\underline{\mu}_{k}^{p}, \underline{\boldsymbol{v}}_{k}\right)\right\}$ depends on the shape matrix $\hat{\mathbf{B}}_{k}^{-1}$ but is independent of the (unknown) measurement source $\underline{z}_{k}$. However, the variance $\operatorname{Var}\left\{\bar{v}_{k}\left(\underline{\mu}_{k}^{p}, \underline{\boldsymbol{v}}_{k}\right)\right\}$ of

\footnotetext{
${ }^{2}$ We observed that for this representation, it may be suitable to multiply the measurement equation with a factor, e.g., $1 / \operatorname{trace}\left(\mathbf{L}_{k} \mathbf{L}_{k}^{T}\right)$, if a Gaussian-assumed estimator is used.
} 


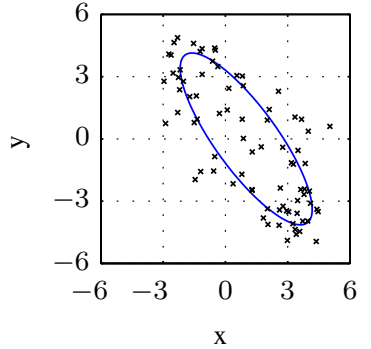

(a) Scenario 1 .

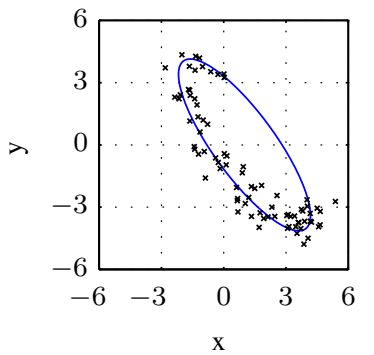

(b) Scenario 2.
Fig. 3: Example measurements received from an ellipse.

$\bar{v}_{k}\left(\underline{\mu}_{k}^{p}, \underline{\boldsymbol{v}}_{k}\right)$ depends on both $\hat{\mathbf{B}}_{k}$ and the vector from the ellipse center to measurement source $\underline{z}_{k}-\underline{\hat{\underline{m}}}_{k}$.

Circle (Center/Radius Representation): An interesting special case is obtained in case of a circle. In this case, (8) becomes

$$
\begin{aligned}
0 & =g\left(\underline{\boldsymbol{p}}_{k}, \underline{\hat{y}}_{k}\right)-\left(\left(\boldsymbol{m}_{k}-\underline{z}_{k}\right)^{T} \underline{\boldsymbol{v}}_{k}+\underline{\boldsymbol{v}}_{k}^{T} \underline{\boldsymbol{v}}_{k}\right) \\
& :=h\left(\underline{\boldsymbol{p}}_{k}, \underline{\boldsymbol{v}}_{k}\right)
\end{aligned}
$$

where $h\left(\underline{\boldsymbol{p}}_{k}, \underline{\boldsymbol{v}}_{k}\right)$ is the new measurement equation with multiplicative noise. Furthermore, the additive noise term in (9) becomes $\bar{v}_{k}\left(\underline{\boldsymbol{p}}_{k}, \underline{\boldsymbol{v}}_{k}\right)=\left(\boldsymbol{m}_{k}-\underline{z}_{k}\right)^{T} \underline{\boldsymbol{v}}_{k}+\underline{\boldsymbol{v}}_{k}^{T} \underline{\boldsymbol{v}}_{k}$. Actually, it turns out that the probability distribution of $\bar{v}_{k}\left(\underline{\mu}_{k}^{p}, \underline{\boldsymbol{v}}_{k}\right)$ does not depend on $\underline{z}_{k}$ in case $\underline{\boldsymbol{v}}_{k}$ is isotropic Gaussian noise. This fact has been shown by the authors in [19], where formulas for the first two moments of $\bar{v}_{k}\left(\underline{\mu}_{k}^{p}, \underline{\boldsymbol{v}}_{k}\right)$ can be found. The major insight in [19] is that in case of isotropic noise, no assumptions on the measurement source has to be made. Note that the measurement equation with additive noise for circles has already been derived (in a different way) in [19] for the special case of isotropic noise.

\section{B. Time Update}

The time update can be performed in the usual manner. The state vector evolves according to a known Markov model characterized by the conditional density function $f\left(\underline{p}_{k} \mid \underline{p}_{k-1}\right)$ derived from the system equation (5). The prediction $f_{k}^{p}\left(\underline{p}_{k}\right)$ at time step $k$ thus results from the Chapman-Kolomogorov equation

$$
f_{k}^{p}\left(\underline{p}_{k}\right)=\int f\left(\underline{p}_{k} \underline{p}_{k-1}\right) \cdot f_{k-1}^{e}\left(\underline{p}_{k-1}\right) d \underline{p}_{k-1} .
$$

In this work, we assume that the predicted density $f_{k}^{p}\left(\underline{p}_{k}\right)$ is approximated with a Gaussian.

\section{Evaluation}

In this section, the new approach for conic fitting is compared to the (Iterated) EKF approach suggested in [2]. In the considered scenario, an ellipse is located at the position $[1,0]^{T}$ in two-dimensional space. The length of the minor axis is 1.5 and length of the major axis is 5 . The

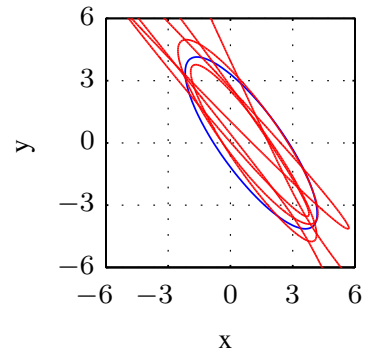

(a) EKF: 30 Measurements.

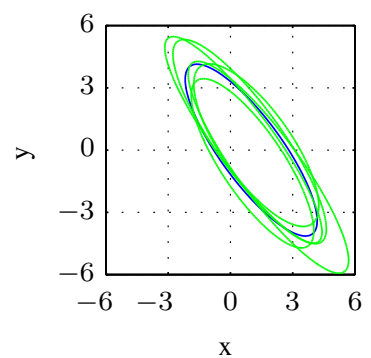

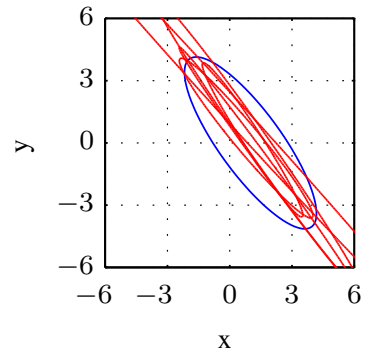

(b) EKF: 80 Measurements.

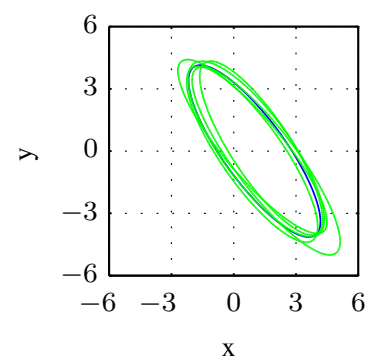

(c) New Method: 30 Measurements.(d) New Method: 80 Measurements.

Fig. 4: Scenario 1: Estimated ellipses from five different runs plotted in one figure.

measurements $\underline{\hat{y}}_{k}$ are received sequentially from the ellipse. We have considered two scenarios. In the first scenario the measurement noise is rather high, i.e., $\Sigma_{k}^{v}=\operatorname{diag}(0.5,0.5)$ (see Fig. 3a) and the measurement sources are uniformly distributed from the ellipse. In the second scenario, the measurement noise is smaller, i.e., $\Sigma_{k}^{v}=\operatorname{diag}(0.2,0.2)$. However, measurement sources are only located on the left side of the ellipse (see Fig. 3b). Both estimators represent an ellipse according to (6). The new estimator is implemented using the measurement equation with multiplicative noise and the UKF [22] for the measurement update. The prior for the ellipse is set to a circle with radius 3 located at the origin, i.e., $\mu_{1}^{p}=[0.5,0,0,0,-4.5]^{T}$. The uncertainty of the prior ellipse is assumed to be very high, i.e., $\Sigma_{1}^{p}=$ $\operatorname{diag}(10,10,10,10,10)$.

For both scenarios, the estimated ellipses for five different Monte-Carlos runs are plotted in Fig. 4 and Fig. 5 after having received 40 and 80 measurements. We observe that for both scenarios, the new method yields much more precise and staple ellipse estimates. The EKF approach in Fig. 4 yields some infeasible estimates that are no ellipses. Furthermore, several estimated ellipses are too small. On the other hand, the new method yields accurate ellipse estimates. In Fig. 6, the root mean squared error (RMSE) for the first 80 time steps demonstrates that the new approach yields more precise estimation results than the EKF, which tends to diverge. 


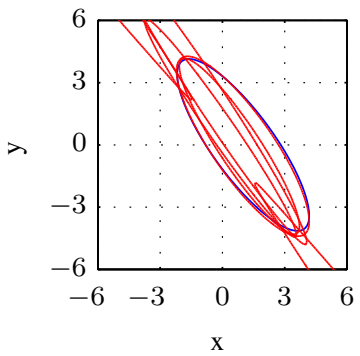

(a) EKF: 30 Measurements.

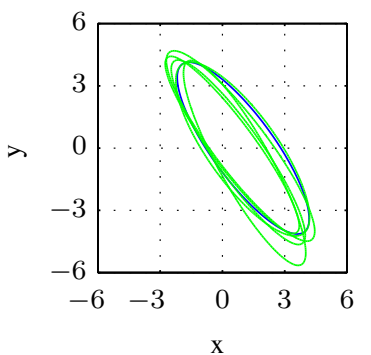

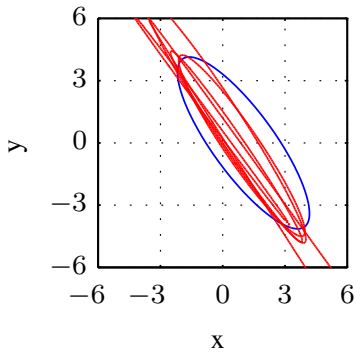

(b) EKF: 80 Measurements.

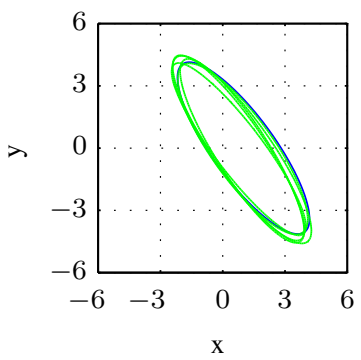

(c) New Method: 30 Measurements.(d) New Method: 80 Measurements.

Fig. 5: Scenario 2: Estimated ellipses from five different runs plotted in one figure.

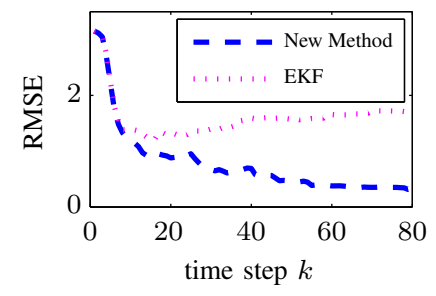

(a) Scenario 1 .

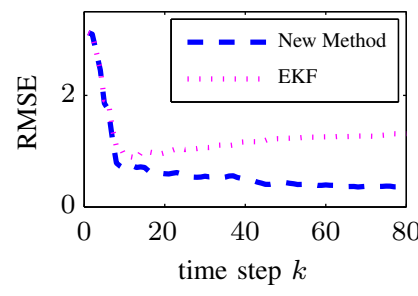

(b) Scenario 2 .
Fig. 6: RMSE plotted over time (averaged over 20 runs).

\section{Vi. CONCLUSion AND Future Work}

In this work, we have considered the problem of fitting a conic to noisy data points in the context of tracking applications. We have presented a procedure for deriving an explicit measurement equation based on the given implicit measurement equation. For the explicit measurement equation, standard Gaussian filters can be used for an efficient recursive measurement update. Future work consists of estimating the noise covariance matrix in addition to the parameter vector.

\section{REFERENCES}

[1] J. Porrill, "Fitting Ellipses and Predicting Confidence Envelopes Using a Bias Corrected Kalman Filter,' Image Vision Computing, vol. 8, pp. 37-41, 1990.

[2] Z. Zhang, "Parameter Estimation Techniques: A Tutorial with Application to Conic Fitting," Image and Vision Computing, vol. 15, no. 1, pp. 59 - 76, 1997.

[3] I. Frosio and N. A. Borghese, "Real-time Accurate Circle Fitting with Occlusions," Pattern Recognition, vol. 41, no. 3, pp. 1041-1055, 2008.
[4] S. J. Ahn, W. Rauh, and M. Recknagel, "Ellipse Fitting and Parameter Assessment of Circular Object Targets for Robot Vision," in Proc. IEEE/RSJ Int. Conf. Intelligent Robots and Systems IROS '99, vol. 1, 1999, pp. 525-530.

[5] N. Greggio, L. Manfredi, C. Laschi, P. Dario, and M. C. Carrozza, "RobotCub Implementation of Real-Time Least-Square Fitting of Ellipses," in Proceedings of the 8th IEEE-RAS International Conference on Humanoid Robots (Humanoids 2008), 2008, pp. 174-181.

[6] N. Greggio, A. Bernardino, C. Laschi, J. Santos-Victor, and P. Dario, "Real-Time 3D Stereo Tracking and Localizing of Spherical Objects with the iCub Robotic Platform," Journal of Intelligent \& Robotic Systems, pp. 1-30, 2011.

[7] P. Nuez, R. Vazquez-Martin, A. Bandera, and F. Sandoval, "An Algorithm for Fitting 2-D Data on the Circle: Applications to Mobile Robotics," IEEE Signal Processing Letters, vol. 15, pp. 127-130, 2008.

[8] S. Zhang, L. Xie, and M. D. Adams, "Feature Extraction for Outdoor Mobile Robot Navigation based on a Modified Gauss-Newton Optimization Approach," Robotics and Autonomous Systems, vol. 54, no. 4, pp. 277 - 287, 2006.

[9] K. O. Arras, Óscar Martínez Mozos, and W. Burgard, "Using Boosted Features for the Detection of People in 2D Range Data," in Proc. IEEE International Conference on Robotics and Automation (ICRA'07), Rome, Italy, 2007.

[10] A. Carballo, A. Ohya, and S. Yuta, "People Detection using Range and Intensity Data from Multi-layered Laser Range Finders," in Proc. IEEE/RSJ International Conference on Intelligent Robots and Systems (IROS), 2010, pp. 5849-5854.

[11] N. Chernov, Circular and Linear Regression: Fitting Circles and Lines by Least Squares. CRC Press, 2010.

[12] D. Hall and J. Llinas, Handbook of Multisensor Data Fusion. CRC Press, May 2001.

[13] A. Fitzgibbon, M. Pilu, and R. B. Fisher, "Direct least square fitting of ellipses," IEEE Transactoins on Pattern Analysis and Machine Intelligence, vol. 21, pp. 476-480, May 1999.

[14] J. Yu, S. R. Kulkarni, and H. V. Poor, "Robust Fitting of Ellipses and Spheroids," in Proceedings of the 43rd Asilomar Conference on Signals, Systems and Computers, 2009, pp. 94-98.

[15] W. Gander, G. H. Golub, and R. Strebel, "Least-Squares Fitting of Circles and Ellipses," BIT Numerical Mathematics, vol. 34, pp. 558578, 1994.

[16] K. Kanatani, "Statistical Optimization for Geometric Fitting: Theoretical Accuracy Bound and High Order Error Analysis," Int. J. Comput. Vision, vol. 80, no. 2, pp. 167-188, 2008.

[17] —, "Ellipse Fitting with Hyperaccuracy," in Computer Vision ECCV 2006, ser. Lecture Notes in Computer Science, A. Leonardis, H. Bischof, and A. Pinz, Eds. Springer Berlin/Heidelberg, 2006, vol. 3951.

[18] M. Werman and D. Keren, "A Bayesian Method for Fitting Parametric and Nonparametric Models to Noisy Data," IEEE Transactions on Pattern Analysis and Machine Intelligence, vol. 23, no. 5, pp. 528534, 2001.

[19] M. Baum, V. Klumpp, and U. D. Hanebeck, "A Novel Bayesian Method for Fitting a Circle to Noisy Points," in Proceedings of the 13th International Conference on Information Fusion (Fusion 2010), Edinburgh, United Kingdom, Jul. 2010.

[20] T. Ellis, A. Abbood, and B. Brillault, "Ellipse Detection and Matching with Uncertainty," Image and Vision Computings, vol. 10, no. 5, pp. 271-276, 1992.

[21] M. Baum, B. Noack, and U. D. Hanebeck, "Extended Object and Group Tracking with Elliptic Random Hypersurface Models," in Proceedings of the 13th International Conference on Information Fusion (Fusion 2010), Edinburgh, United Kingdom, Jul. 2010.

[22] S. J. Julier and J. K. Uhlmann, "Unscented Filtering and Nonlinear Estimation," in Proceedings of the IEEE, vol. 92, no. 3, 2004, pp. 401-422.

[23] D. Simon, Optimal State Estimation: Kalman, H Infinity, and Nonlinear Approaches, 1st ed. Wiley \& Sons, August 2006.

[24] M. Baum, B. Noack, F. Beutler, D. Itte, and U. D. Hanebeck, "Optimal Gaussian Filtering for Polynomial Systems Applied to Associationfree Multi-Target Tracking," in Proceedings of the 14th International Conference on Information Fusion (Fusion 2011), Chicago, Illinois, USA, Jul. 2011.

[25] R. Kan, "From Moments of Sum to Moments of Product," Journal of Multivariate Analysis, vol. 99, no. 3, pp. 542-554, March 2008.

[26] K. B. Petersen and M. S. Pedersen, "The matrix cookbook," 2008. 